\title{
Survey on Content Prefetching for Mobile User from Cloud with less Energy Conserving Transmission Protocol
}

\author{
Nitin R. Kankure ${ }^{1}$, Vina M. Lomate ${ }^{2}$ \\ ${ }^{1}$ ME. Student, Dept. of Computer Science, RMD Sinhgad School of Engineering, Savitribai Phule Pune University, Pune, India \\ ${ }^{2} \mathrm{HOD}$ of the Computer Engineering Department, RMD Sinhgad College of Engineering Savitribai Phule Pune University, Pune, India
}

\begin{abstract}
Now in the $21^{\text {st }}$ century every individual person has smart phone so everyone want to access the data in the form for news, blogs, job portals updates, current affairs Multimedia data in the smart phone by the use of internet of thing.So to do this user has to install all kind different application available in play store market By use of this application user will access all kind of data but by doing this user smart phone consume lot of energy for fetching sending data to server, so in this paper by the use of cloud computing technology we propose new method for fetching data for user application for news blog,current affairs, job alert, in one application by use of less energy conserving transmission protocol In this methodology consist of two part i.e RSS Content Perfecting for all Subscribed mobile user from server and pushing data back to user by knowing wireless link capability of user by use of with less Energy Conserving Transmission protocol Due to this methodology large amount of traffic reduce on server for data transmission And we will avoid redundant downloading of data.
\end{abstract}

Keywords: Cloud Computing, RSS, Prefetching, Pushing

\section{Introduction}

Now a days use mobile devices is more in our day to day life user will use mobile device for accessing social network application(Facebook, twitter ,LinkedIn ),reading all new papers, various blogs online ,keeping in touch with current affairs in one touch so the use of mobile smart phone increasing day by day in user life ,However, mobile devices are not without drawbacks. First, the processing power of these devices is necessarily less than that of traditional computers. This is not only because of the small size of mobile devices, but also because of the lack of cooling for hardware components and the wireless link capacity cannot keep up with the Huge traffic demand. The gap between the traffic demand and the connection capacity, along with timevarying connection conditions, results in poor service quality of mobile services over current cellular networks $(3 \mathrm{G} / 4 \mathrm{G})$, such as long loading/buffering time and intermittent disruptions. Therefore, the main study/Research fall in the following three issues[2].

1)How to reduce the traffic load via the Internet regarding many mobile users?

2)How to Save the power energy of mobile devices?

3) How to guarantee the quality of service of user Demands [2]

This paper is prepared as follows. Basic Working of RSS Feed, Cloud Computing Technology, Prefetching and Pushing Methodology in Section 2. Section 3 illustrates the Proposed System For Problem Statement. Section 4 explains the existing method and Related Work and Section 5 includes the proposed method and System Architecture. The final section provides a conclusion for our Paper Title.

\section{Literature Survey}

\subsection{RSS feed}

RSS (Really Simple Syndication) is a format for delivering frequently varying web content. Many news related sites, web Content and other online publishers organized their content as an RSS Feed to whoever wants it. RSS solves a problem for User who regularly uses the web for updated content. It allows user to easily stay informed by retrieving the Recent Updated content from the sites you are interested in. Mobile User saves time by not needing to visit each site individually again and again. You ensure your privacy, by not need to join each site's RSS Feed subscription every time. The number of Sites offering RSS Feeds is increasing day by day rapidly and includes names like Yahoo News, Google News

\subsection{Cloud Computing}

The practice of using a network of remote servers hosted on the Internet to Store, Retrieve, manage Data, and process data, rather than a local server or a personal computer. it has always been divided into three service categories: infrastructure as a service, platform as a service and software as service. 


\section{International Journal of Science and Research (IJSR) \\ ISSN (Online): 2319-7064}

Index Copernicus Value (2013): 6.14 | Impact Factor (2014): 5.611

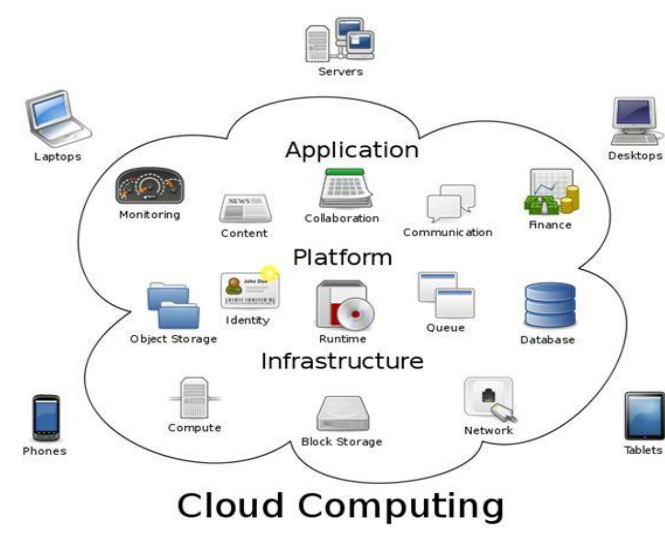

Figure 1: Cloud Computing Architecture

\subsection{Prefetching}

Perfecting is simply loading/Download a Required file before it's needed to user. It is common on Web sites for a Mobile user action to download of additional data, such as updated news, web content or images. If it is possible to predict the next user Task, then it may be possible to start the downloads that Action before the user input is made

\subsection{Pushing}

Transmitting data from an local internal network or a cloud based service to the user's Personal computer or mobile Smart phone device. Updated News feed, Web Content and text messages are pushed in real time, whereas e-mail and calendar updates are usually pushed at intervals of time. Cloud Server based services push User personal photos, videos and documents to all their devices to keep them in sync to server in online mode. Regular updates to applications are also a form of push technology

\section{Proposed System}

In this Given paper, we Explained two methodologies first one for content perfecting for Mobile User from cloud with the use of RSS Feed

RSS is an extensive markup language (XML) application, and the idea behind RSS is to enable users to be informed when the Content on the Internet has changed. As the concise RSS formats allow relatively low-bandwidth data Collection even for several different source, RSS is becoming a mobile Phone method for notify the users of new news update, content, particularly in frequently updated websites such as Web blogs and news portals site .For receiving RSS-based Information Content, the RSS feed could contain one or more channels for one RSS document [2]. The RSS channels and items are composed of a Content title, a description for that content, Content URL, and a publishing date of document, the abstract of the document for Feed. The mobile users can easily access and categorize the received contents with the abstract information of web content, and then the full version of the update can be further Retrieved, including multimedia files (e.g., images and videos), while providing RSS feeds with many subscribed user, if one Mobile user Fetch the RSS updates from server and thus the attached content files for same, multiple users will induce multiple downloads of the same web content files via the Internet[2]; hence, there is the significant redundancy downloading problem, Therefore, in order to avoid unnecessary data content delivery from the RSS feed service providers to the user, So we will make a clustering point on server with data caching functionality to gather RSS updates with content files, and once duplicated requests come, the stored cached content can be directly utilized without Downloading again[2]
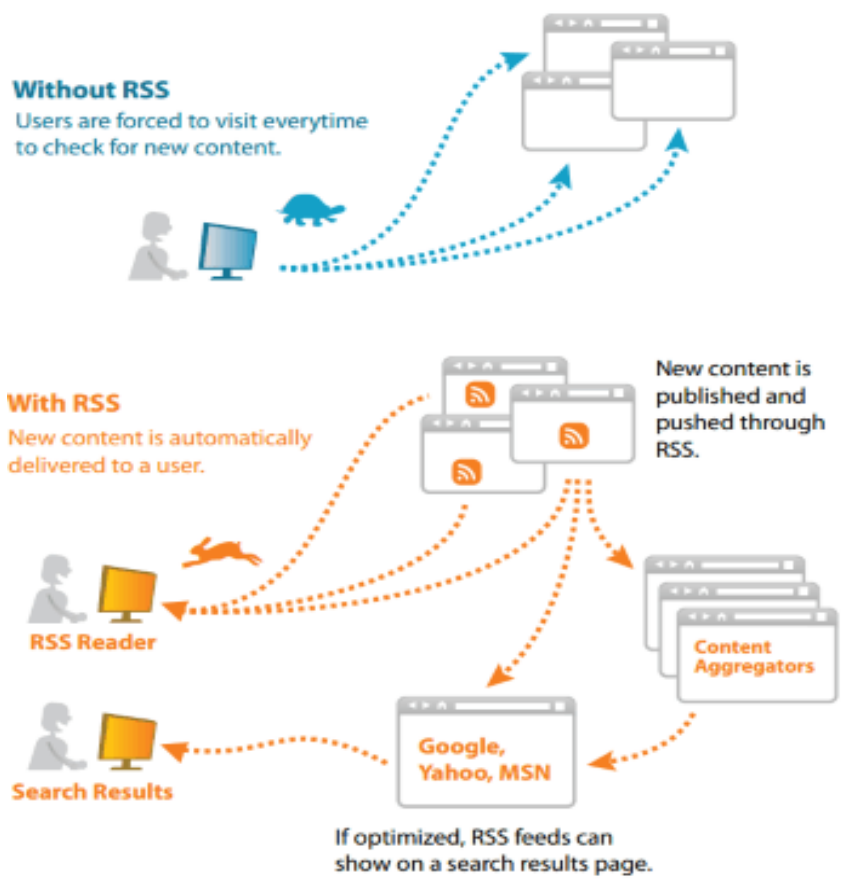

Figure 2: RSS Feed Concept/Working

Second one for less Energy transmission during data transfer, an Application-layer Adaptive Transmission Protocol targeting at energy-efficient data transfers between mobile devices and the cloud platform. The Advantage of this approach is hinged upon two observations: First, the energy consumption in transmission is largely affected by the intrinsic stochastic nature of wireless networks [1]. This is especially true due to the instability of wireless connections and the fluctuation of communication bandwidth. Measurements show that more energy is consumed during bad Signal connectivity, while low energy is consumed with good connectivity in mobile devices [2]. Second, many mobile applications are delay-tolerant, such as music/video download and Web data backup from server, where as many other tasks are prefetch friendly, for example, Information retrieval in social networking sites such as Twitter and Facebook

\section{Related Work}

Therefore, Inspired by the trend of mobile phone RSS reading and the fast development of cloud computing server, in this paper, we propose various Method to improve the quality of RSS service for mobile users, consisting of following two parts: 


\section{International Journal of Science and Research (IJSR) \\ ISSN (Online): 2319-7064}

Index Copernicus Value (2013): 6.14 | Impact Factor (2014): 5.611

\subsection{Cloud-Guided Prefetching}

Which will periodically fetch the multimedia content Information of the RSS feeds for all subscribed mobile users from the Cloud server[2]

\subsection{Data Pushing}

This will push the web content information, or a part of the web content information, to mobile users at an suitable time by evaluating the bandwidth link quality of mobile, energy consumption, and user Quality Of Service requirements [2].

We list down the main features and the corresponding contributions of framework as follows.

1)In this, users first share their subscribed RSS lists to the cloud agent, which will minimize the duplicated RSS

(Due to the difference of the RSS popularity)[2].

2)Depending on user activity profile, the cloud agent will assign a specific Task for periodically updating the RSS feed list to obtain new published web content with proper interval of time to the mobile user.

3)From the RSS, after the cloud agent Get the abstracted information of the content from source, it will further fetch the content by parsing the XML web content data and fetch the original multimedia files (usually from HTML websites) and then store the obtained data in forms of texts, images, and videos in the server locally[2].

4) The cloud agent will check the link quality of wireless data bandwidth and mobile activities of users, as well as the usage condition, so that an appropriate time will be chosen for pushing the content to the mobile devices cognitively.

5)Based on Above type of implementation and related evaluation, cloud center can avoid duplicated [2] Downloads and reduce the total traffic load up to $60 \%$.

\section{System Architecture}

The System Architecture Consist of following parts

\subsection{Mobile Application}

Use will register/installed this application in mobile and this application will have access to all subscribed feed of user .mobile application will communicate to cloud Server via service API in middle layer

\subsection{Service API}

This is middle layer of application in the client and server communication this service API get request from client and send client request to the cloud server for data request /response

\subsection{Cloud Server}

In this cloud server contains following parts

\subsubsection{RSS Feed}

This RSS feed contains the all the RSS feed subscribed to user in server it will get the updated RSS feed from the resource fetcher and store all RSS feed in database and send back RSS feed to End User on the client request.

\subsubsection{Resource Fetcher}

This component is responsible for all interaction with Content sources it will fetch all the content from the web site for the user request and store the data content in the database and RSS feed for future retrieval of data

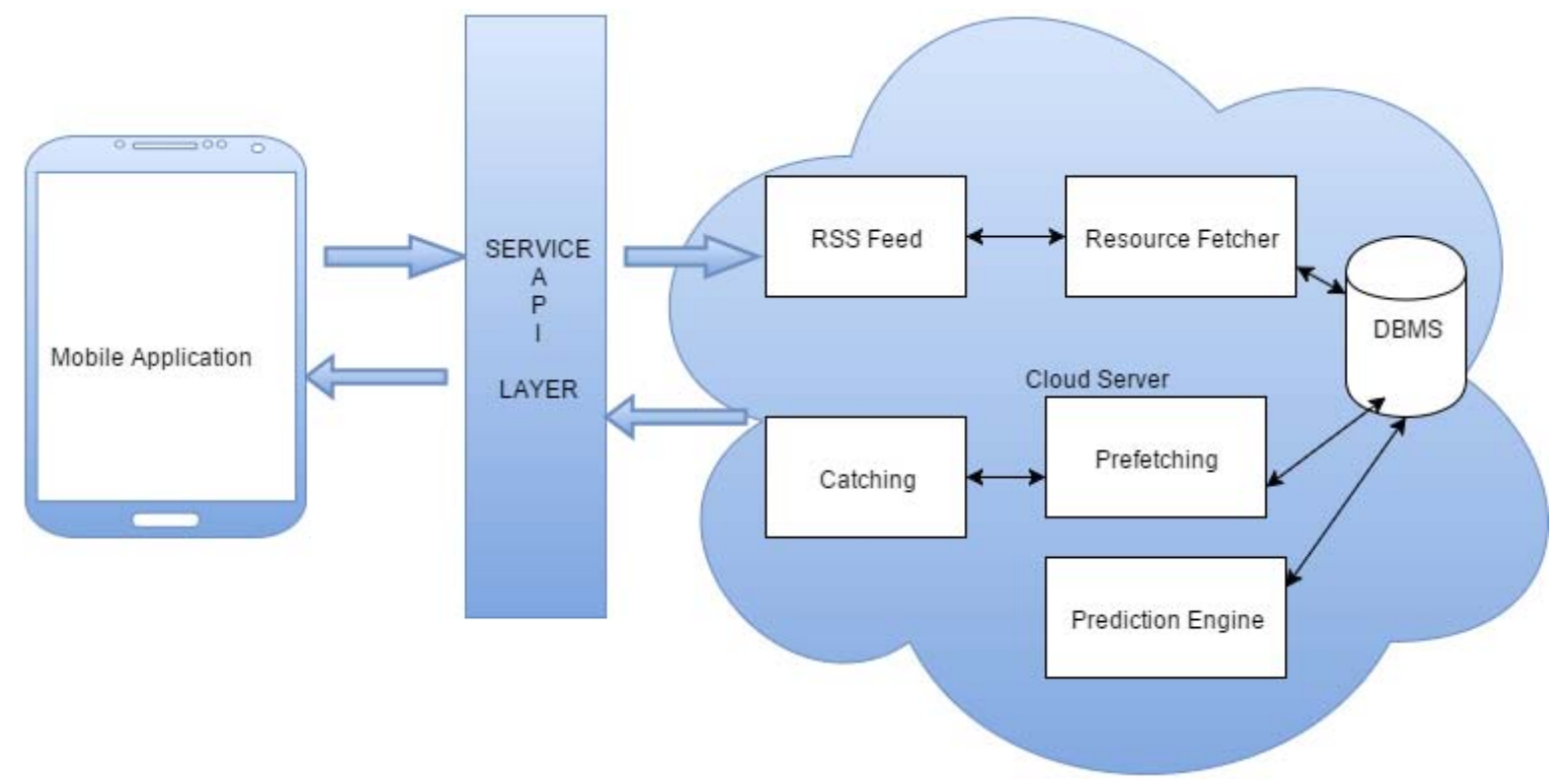

Figure 3: System Architecture

\subsubsection{Prediction Engine}

The Prediction Engine is responsible for suggesting items of content that are likely to be accessed by a particular user in the near future. It is therefore where all data analysis takes place. In order to accomplish this task, the
Prediction Engine is informed of all user activity by the Resource Server from mobile application. The Prediction Engine is also provided the requested content itself, although it may or may not make use of this information. For example, in an implementation that is intended to apply to multiple application domains, a prediction engine would most likely 


\section{International Journal of Science and Research (IJSR) \\ ISSN (Online): 2319-7064 \\ Index Copernicus Value (2013): 6.14 | Impact Factor (2014): 5.611}

process user request metadata but choose to ignore the content of these requests, as the structure of this content is likely to vary across different domains.

\subsubsection{Catching}

The Cloud Cache and Mobile Cache are to be implemented by the application developer. The cloud cache stores preprocessed, pre cached content accessible by all users. The mobile caches on the other hand store processed, pre fetched content specific to the user of the resident device. Both cloud and mobile caches can be implemented with unique policies to satisfy unique usage patterns, making the middleware as general as possible. In this way, the caches can be optimized with contextual knowledge from the mobile device, and auxiliary information from the prediction engine

\subsubsection{Prefetching}

Prefecting is simply loading/Download a Required file before it's needed to user. It is common on interactive sites for a user action to download of additional data, such as updated news, web content or images. If it is possible to predict the next user action, then it may be possible to start the downloads before the user input is made

\section{Conclusion}

In this paper, we have used the cloud computing technology to propose a new framework to improve the quality of RSS reading service for mobile user. this framework consists of two parts, i.e., cloud-assisted data Pre fetching, which will proactively fetch the multimedia data content of the RSS feeds for all subscribed mobile users, and periodically pushing, which will push the web content to mobile users at an appropriate time by evaluating the mobile bandwidth link quality and user Quality Of Service requirements. Furthermore, the social impact among users is considered. We will implement a Application for this above prototype for Getting all feeds in one application and evaluate performance of application in terms of Energy consumption required by that application.

\section{References}

[1] Fangming Liu, Member, IEEE, Peng Shu, and John C.S. Lui, Fellow, IEEE" AppATP: An Energy Conserving Adaptive Mobile-Cloud Transmission Protocol" IEEE TRANSACTIONS ON COMPUTERS, VOL. 64, NO. 11, NOVEMBER 2015

[2] Xiaofei Wang and Min Chen," PreFeed: Cloud-Based Content Prefetching of Feed Subscriptions for Mobile Users" IEEE SYSTEMS JOURNAL, VOL. 8, NO. 1, MARCH 2014

[3] Wai Yip Lum and Francis C.M. Lau, Member, IEEE Computer Society "User-Centric Content Negotiation for Effective Adaptation Service in Mobile Computing" IEEE TRANSACTIONS ON SOFTWARE ENGINEERING, VOL. 29, NO. 12, DECEMBER 2003

[4] C. Lai, H. Chao, Y. Lai, and J. Wan, "Cloud-assisted real-time transrating for http live streaming," IEEE Wireless Commun., vol. 20, no. 3, pp. 62-70, Jun. 2013
[5] S. Zhao, P. P. C. Lee, J. C. S. Lui, X. Guan, X. Ma, and J. Tao, "Cloud based push-styled mobile botnets: A case study of exploiting the cloud to device messaging service," in Proc. ACSAC, Dec. 2012, pp. 119-128

[6] K. Kumar and Y. Lu, "Cloud computing for mobile users: Can offloading Computation save energy?" IEEE Compute., vol. 43, no. 4, pp. 51-56, Apr. 2010

\section{Author Profile}

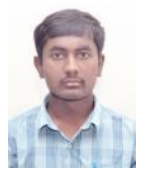

Nitin R Kankure Research Scholar RMD Sinhgad of Engineering, Savitribai Phule Pune University. He Received B.E. in Information Technology from Savitribai Phule Pune University. Currently he is pursuing M.E. in Computer Engineering from RMD Sinhgad School of Engineering, Warje, Savitribai Phule Pune University, Pune

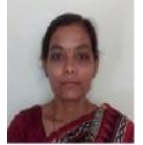

Prof. Vina M. Lomte is the HOD of Computer Dept. at RMD SSOE College, Pune, having more than $10+$ years of experience in the field of teaching and research. The domains of her research are Software Testing, Software Engineering and Web Security. 